\title{
IVF AND ET: CAN IT EVER BE PATIENT-FRIENDLY AND AFFORDABLE?
}

\author{
${ }^{*}$ M. O. Aziken, ${ }^{*}$ O. O. Kalu \\ *Department of Obstetrics and Gynaecology, University of Benin Teaching Hospital, \\ Benin City, Nigeria.
}

\section{Correspondence:}

Dr M. O. Aziken

Department of Obstetrics and Gynaecology,

University of Benin Teaching Hospital,

P.M.B. 1111 Ugbowo

Benin City, Nigeria

Email: michaelaziken@yahoo.com

\begin{abstract}
Whilst considered a routine procedure today, in vitro fertilization (IVF) still face huge opposition with cost consideration being an important impediment to its widespread acceptance. Various interventions to make IVF more patient friendly and cost- effective involve proper patients' education, modifications in ovulation induction, egg retrieval, embryo incubation, and luteal phase support protocols.
\end{abstract}

\section{INTRODUCTION}

The story of in vitro fertilization (IVF) has its beginning in 1973 with a lady, Doris Del-Zio, and her struggle to have a child. ${ }^{1}$ Desperate following multiple failed attempts to correct her blocked fallopian tubes, Del- Zio agreed to undergo an experimental procedure that had hitherto only been tested on lower mammals. Following surgical removal of a few eggs from her and fertilization of same on a glass test-tube with the resulting zygote cultured in a medium, the covert procedure was discovered and shut down by the boss of the eccentric researcher Landrum Shettle of the Columbia University. Shettles' boss, Raymond Vande, did so for fear of the reputation of the school. The distraught Zio and her husband frustrated at this loss of opportunity sued. ${ }^{1}$

The opportunity for the first successful IVF expectedly occurred in Europe as the prevalent thinking then across North America was that IVF would lead to genetic engineering and selective breeding, which would destroy the concept of humanity. ${ }^{1,2}$ The birth of Louise Brown on the $25^{\text {th }}$ of July, 1978 from the historic collaboration between Robert Edwards, a scientist from Cambridge, and Patrick Steptoe, a Gynaecologist in Lancashire, announced the first birth from IVF and heralded a new dawn in the management of infertility. ${ }^{1,3,4,5}$ The IVF was performed because Lesley Brown had no tubes (which had been removed from two previous ectopic) but had normal uterine cavity and was normally ovulating. ${ }^{4}$

Since this landmark event, achieved from a natural cycle, the procedure of IVF has undergone several modifications with the aim of improving success rate (human reproduction itself being a notably wasteful process). ${ }^{3,4,5}$ While the basic principles of oocyte collection, extra-corporal fertilization and subsequent intrauterine transfer of the resulting embryo have remained the same, ${ }^{3}$ many modifications of the original technique has however been introduced over the 
years to improve the result of treatment and patient acceptability of the procedure. These modifications have on the other hand resulted in an increased cost of the procedure and resultantly hampered patients' acceptance and ability to undergo the procedure.

Cost is a major obstacle for most patients. ${ }^{3,6}$ Treatment involves injections of expensive medications; monitoring ovarian response to medication with serum blood tests such as estradiol $\left(E_{2}\right)$, luteinizing hormone (LH), and progesterone $\left(\mathrm{P}_{4}\right)$; monitoring follicular development with pelvic ultrasound examination; egg retrieval; fertilization of egg and the embryo culture; and, finally, embryo transfer. If any embryo remains after transfer is completed, there can be the additional cost of freezing and storing the spare embryos ${ }^{6,7}$. IVF is also physically and emotionally intense ${ }^{6}$. Hence, despite proven success and benefits, IVF remains unavailable to many. ${ }^{6}$ An ideal technique therefore would be one with a protocol that is highly effective and yet reduces expense and increases patients' acceptance. Cost reduction is especially needed in a resource-limited setting.

Despite these impediments, IVF clinics are found in almost every country worldwide $^{6}$, and IVF is used to treat many aetiological factors in infertility including tubal diseases, male factor, endometriosis, and unexplained infertility ${ }^{3,5,6}$. With this increasing popularity, the need arises to explore ways of tackling cost and other impediment to patients' acceptance of the procedure. This review attempts to highlight current standard protocol of IVF regimen while exploring some of the area where cost-saving and patient friendly modifications have been employed by workers in different settings. It is hoped that wider adaptation of some of these modifications would go a long way in making the process of IVF more accessible and acceptable to the would-be patient.

\section{Patient Education}

Patient counselling is a vital part of initial and subsequent patient care. Patients are expected to master a lot of new information; they need to understand the steps involved in the process as well as learn how to administer injections. This typically may take upwards of an hour meeting ${ }^{6}$ and may be more time-consuming for the semi-illiterate patients that may be seen in resource-limited settings. Many clinical settings have developed special multi-media education systems to provide IVF orientation. This typically involves video clips that last 25-30 minutes, each programmed to meet each couples need. This multimedia education may be provided on group basis to patients on initial contact or in a 'batch' of selected patients. Further questions and individual concerns can then be taken privately to meet a couples' particular need. The multimedia video clip has the unique advantage of ensuring consistency of information disseminated. ${ }^{6}$

\section{Ovarian Stimulation:}

One difficult task faced by patients on IVF treatment is learning to perform intramuscular (IM) injections required as part of IVF ovarian stimulation protocol. Although self-administration is possible, it is common to conscript a second partner, usually a spouse. Getting a willing party that will be available at all times to partake in injection administration can be a source of stress, as the party needs to be available at all times and needs to be trained in the technique of injection administration. To subvert this 
problem, subcutaneous (SC) injection is increasingly being embraced because of ease of self-administration and easier tolerability.

Controlled ovarian stimulation in IVF typically involves the use of gonadotropin releasing hormone agonists (GnRH-a) and follicle stimulating hormone (FSH). The $\mathrm{GnRH}-\mathrm{a}$ is used to achieve pituitary down-regulation, prevent premature ovulation and stimulate the development of several mature follicles from the ovary and increase the chances of obtaining fertilisable eggs. ${ }^{3,4,6}$ Typically, twice daily injections may be required to be given. Reducing the number of injections from four to two or from two to one (by mixing the $\mathrm{GnRH}-\mathrm{a}$ into prepared vial of $\mathrm{FSH}$ ) has been found to reduce injection related anxiety of even the least 'needle-phobic' patient without causing any clinical difference in the number of eggs retrieved and fertilized or on the quality of embryos and pregnancy rates $^{6}$ - although local reactions are commoner with SC injections compared to IM injections.

Long protocols are still at present the most widely used protocols throughout the world ${ }^{5}$ and involve the use of a $\mathrm{GnRH}-\mathrm{a}$ (taken on a daily basis nasally \{e.g Buserelin, Naferelin\}, or daily subcutaneous injections $\quad$ e.g Buserelin, Leuprorelin\} or in a depot preparation \{Goserelin, Leuprorelin\}) to achieve pituitary down-regulation before administration of gonadotrophins. Typically pituitary desensitization using $\mathrm{GnRH}-\mathrm{a}$ is commenced between days 19 and 22 of the preceding cycle (rationale being to create a temporary menopause from which the ovaries can be stimulated by use of $\mathrm{FSH} / \mathrm{hMG}$ ). Following confirmation of adequate suppression (by TVS and/ or serum estradiol measurement), done usually 7-10days after initiation of $\mathrm{GnRH}-\mathrm{a}$, the gonadotrophins are started until adequate ovarian response- presence of 2 or more follicles $\geq 18 \mathrm{~mm}$.

Short (flare) protocols have also been developed and may be agonist or antagonist protocols. The short agonist protocol involves starting $\mathrm{GnRH}-\mathrm{a}$ on day 2 and $\mathrm{FSH} / \mathrm{hMG}$ on day 3of the menstrual cycle and is used for those whom difficulty with ovarian stimulation is anticipated- those $\geq 40 y e a r s$, those with high initial $\mathrm{FSH}$ levels and those with prior failure with the long protocol. More recently, a more patient friendly $\mathrm{GnRH}$ antagonist protocol (using Ganirelix and Cetrorelix) has been introduced. . $^{3,6}$ The antagonist has an almost immediate effect on the pituitary and does not need several days to achieve menopausal levels that agonists do. A daily SC injection of $0.25 \mathrm{mg}$ Cetrolelix is normally given, although there is a $3 \mathrm{mg}$ dose whose effect can last for several days. This is commenced on first day of $\mathrm{FSH} / \mathrm{hMG}$ stimulation (usually starting from the fifth day or when the lead follicle is a certain size on USS $\{14 \mathrm{~mm}\}$ and continued alongside the $\mathrm{FSH} / \mathrm{hMG}$ stimulation until adequate response and then stopped prior to hCG injection. The benefits of antagonists over agonists include no menopausal side effects, no cyst formation from initial gonadotrophin surge, shorter cycle duration, less gonadotrophin required per cycle $^{5}$ - and therefore lower drug cost! To circumvent high cost of drugs and promote patient convenience, there is a place for the use of other treatment protocols to achieve superovulation while avoiding the disadvantage of early Luteinizing Hormone (LH) surge. One such protocol is the low cost Clomiphene Citrate/Human Menopausal Gonadotrophin (hMG) protocol which has been noted to achieve similar clinical pregnancy rates as the standard Buserelin/hMG protocol. ${ }^{8}$ 
Use of clomiphene citrate instead of FSH for ovarian stimulation significantly reduces cost, significantly reduces the duration of treatment, produces satisfactory pregnancy rates and reduces the number of injections a patient receives. ${ }^{8}$

There is also the stop protocol wherein the $\mathrm{GnRH}-\mathrm{a}$ is stopped on start of hMG administration.

Following the $\mathrm{GnRH}-\mathrm{a}$ and $\mathrm{FSH}$ administration ovulation is triggered by administration of 10,000iu human chorionic gonadotrophin (hCG) $(5,000 i u$ for those at risk of ovarian hyperstimulation syndrome \{OHSS\}) when two or more follicles measuring $18-20 \mathrm{~mm}$ in diameter is noted.

\section{Egg Retrieval:}

Retrieval of oocytes during IVF is uncomfortable. Various ways of inducing analgesia and anaesthesia have been described, including general anaesthesia, oral narcotics, paracervical block and regional anaesthesia. Paracervical block, as employed in our setting, and use of short acting general anaesthetic agents may be ideal as they enhances full recovery and allows for quicker discharge patients.

Oocyte retrieval was done laparoscopically in the past but the advent of real-time USS has allowed a less invasive USS-directed needling of the ovaries. The laparoscope is occasionally used if the ovaries are inaccessible transvaginally.

The follicular aspiration is scheduled for 34-36 hours after hCG injection prior to physiological ovulation occurring. If hCG is incorrectly administered, few or no eggs are obtained at egg collection itself.

Eggs are fertilized either by routine insemination of approximately 50,000 to 100,000 normally motile sperm or by ICSI.

\section{Embryo Incubation:}

The process of embryo incubation is an exacting one that requires the services of an embryologist. A variety of media have been used with success for embryo culture. Electrolyte concentrations are usually adjusted to simulate the levels in a normal human Fallopian tube. Not only is the temperature carefully controlled in the incubator, but also the gas content and $\mathrm{pH}^{7}$

It is unfortunate that many centres in our setting still rely on the services of foreign embryologists to handle the laboratory aspects of IVF rather than focus on training (formal or on-hands) of local staffs. The cost of catering for these foreign personnel adds significantly to the high cost of IVF and centres in our setting may do well in embarking on training of selected staffs in particular aspects of embryology.

\section{Post embryo Transfer:}

There is no evidence to support the varied instructions given to patient that have undergone IVF post-embryo transfer; there is perhaps more myth in these than in any other aspect of IVF. Some programmes require patients to remain on strict bed-rest for nearly 2 weeks from embryo transfer until pregnancy test, even when there are no data to support such restriction. Because many patients may be anxious and express caution, it may be ideal to allow the patients to rest for anywhere up to 2 hours, if anything, to act as a source of psychological support.

\section{Luteal Phase Support:}

Whilst pregnancies resulting from natural IVF cycles do not require luteal phase supplementation, with the use of $\mathrm{HMG} / \mathrm{GnRH}-\mathrm{a}$ or $\mathrm{GnRH}$ - antagonist it is necessary to support the luteal phase with hCG or progesterone the 
basis being that super-ovulation may impair normal corpus luteal function. ${ }^{5}$ Use of hCG for luteal support is associated with a higher risk of ovarian hyperstimulation than progesterone. Progesterone has the added advantage of coming in different formulations (tablets, injections, vaginal pessaries and rectal suppositories) and this may further optimize patients' satisfaction.

\section{CONCLUSION}

Over the last 25 year the success rate and types of IVF have greatly improved and at present there are well over 2 million babies born throughout the world by this technique. ${ }^{5}$ However, only a very small percentage of patients that require this form of 'terminal care' are able to access it. Unlike in the past when opposition to the then novel concept by different strata of society ${ }^{9}$ had defined acceptance, presently major impediment to acceptance and access to IVF are the cost of treatment and patients' satisfaction. Practicable measures to optimize these at different levels of the IVF process have been highlighted. Cost consideration is especially important in resourcelimited settings where, though infertility is widespread and a serious concern especially to the woman leading on to marital disharmony, neglect, abuse, loss of social security, polygamy and even suicide, ${ }^{4}$ the case that had been made for a redirecting of attention by assisted conception practitioners, from IVF to the preventive aspects of infertility management on the fear that extending infertility treatment to include ART may be impracticable. ${ }^{10,11,12}$

\section{REFERENCES}

1. Mosier E. Book Review: Opening Pandora's Baby. Interface Net Zine. Issue 2, Spring 2005. New
York: Houghton Mifflin Company. 2004.

2. Dutney A. Religion, infertility and assisted reproductive technology. Best Practice and Research Clinical Obstetrics and Gynaecology 2007; 21(1): 169-180. http://science direct.com

3. Ajayi AA. Advances in Assisted reproduction. Archives of Ibadan Medicine. Vol 3, No. 1. 9-12.

4. Orhue AAE. Assisted Reproduction Technology in a Resource Limited Setting. BJPM 2008; 10(1):20-33.

5. Trew G. Assisted reproduction In: Dewhurst's Textbook of Obstetrics and Gynaecology $7^{\text {th }}$ edition. Ed Edmonds K. Blackwell Publishing. 461-478.

6. Alper MM. The Patient-Friendly IVF Cycle. Reproductive Technologies Volume 10, Number 3. 122-125.

7. Holzman GB. (ed). Assisted Reproductive Technology In: Precis, reproductive endocrinology: an update in obstetrics and gynaecology. A.C.O.G. 1998.92103.

8. Onuh SO. Low-cost Clomiphene citrate/ Human Menopausal Gonadotropin (hMG) Protocol for In Vitro Fertilization/ Intra-Cytoplasmic Sperm Injection Achieves Similar Clinical Pregnancy Rates as the Standard Buserelin/hMG Regimen. Being an abstract of the presentation at the Nigerian Fertility Society (NFS) Conference, 2005.

9. Dutney A. Religion, infertility, and assisted reproduction technology. In: Best Practice and Research Clinical Obstetrics and 
Gynaecology. Elsevier 2007; 21(1): 169-180.

10. Okonofua FE. The case against new reproductive technologies in developing countries. Br. J. Obstet 1996: 957-967.
11. Sheth SS. Malpani A.N.: Inappropriate use of New Technology: impact on women's Health. Int. J. Gynaecol. 1979: 58: 159-165.

12. Okonofua FE. New Reproductive technologies and infertility treatment in Africa (Editorial) Afr. J. Reprod. Health 2003: 7. 